\title{
Relación entre las fases precoces de la enfermedad renal y el síndrome metabólico
}

\author{
Manuel F. Landecho ${ }^{\mathrm{a}, *}$, Inmaculada Colina ${ }^{\mathrm{a}}$, Ana Huerta ${ }^{\mathrm{a}, \mathrm{b}}$, Ana Fortuño ${ }^{\mathrm{b}}$, Guillermo Zalba ${ }^{\mathrm{b}}$ \\ y Oscar Beloqui ${ }^{\mathrm{a}}$ \\ a Departamento de Medicina Interna, Clínica Universidad de Navarra, Pamplona, Navarra, España \\ b Área de Ciencias Cardiovasculares, Centro de Investigación Médica Aplicada (CIMA), Universidad de Navarra, Pamplona, Navarra, España
}

Historia del artículo:

Recibido el 31 de mayo de 2010

Aceptado el 26 de noviembre de 2010

On-line el 9 de abril de 2011

\section{Palabras clave:}

Enfermedad renal precoz

Resistencia a la insulina

Síndrome metabólico

Arteriosclerosis subclínica

\begin{abstract}
R E S U M E N
Introducción y objetivos: La enfermedad renal avanzada es un importante problema sanitario por su alta morbimortalidad cardiovascular. La detección y el tratamiento precoces podrían ayudar a evitar su progresión. El síndrome metabólico y la insulinorresistencia se han implicado en la patogenia del daño renal avanzado. Nuestros objetivos son estudiar la relación entre la enfermedad renal precoz (ERP), el síndrome metabólico y la insulinorresistencia y valorar su asociación con marcadores de arteriosclerosis subclínica.

Métodos: El estudio incluyó a 1.498 pacientes. La insulinorresistencia se definió como HOMA $\geq 3,7$ mmol $(\mu \mathrm{U}) / \mathrm{I}^{2}$ y la ERP, como los estadios 1 y 2 de la NKF-KDOQI. Se utilizó el grosor íntima-media carotídeo como marcador de arteriosclerosis subclínica.

Resultados: La presencia de un componente de síndrome metabólico se asoció con una odds ratio (OR) de ERP de 2,3 (intervalo de confianza [IC] del 95\%, 1,18-4,48), que aumentó hasta 6,72 (IC del 95\%, $3,56-13,69$ ) en sujetos con el síndrome. Todos los componentes salvo el bajo valor de lipoproteínas de alta densidad se asociaron con OR elevada de ERP. El incremento del HOMA también se asoció en relación directa con la probabilidad de ERP, que llegó a OR = 3,89 (IC del 95\%,1,99-7,59) para los sujetos del cuarto cuartil. Los sujetos con síndrome metabólico y ERP mostraron un grosor íntima-media carotídeo aumentado frente a aquellos sin enfermedad renal.

Conclusiones: La insulinorresistencia, el síndrome metabólico y sus componentes, salvo bajo valor de lipoproteínas de alta densidad, se asociaron significativamente con OR aumentada de ERP. El síndrome metabólico y la ERP se asociaron de forma independiente y aditiva con arteriosclerosis subclínica. (c) 2010 Sociedad Española de Cardiología. Publicado por Elsevier España, S.L. Todos los derechos reservados.
\end{abstract}

\section{Connection Between the Early Phases of Kidney Disease and the Metabolic Syndrome}

\section{A B S T R A C T}

Introduction and objectives: Advanced kidney disease is a major health problem due to its association with high cardiovascular morbidity and mortality. Early recognition of advanced kidney disease is the mainstay to avoid its progression. Since metabolic syndrome and insulin resistance are risk factors for both cardiovascular and advanced kidney disease, we investigated the relationship of early kidney disease (EKD) with metabolic syndrome and insulin resistance, and their association with surrogate markers of arteriosclerosis.

Methods: We studied 1498 subjects. Insulin resistance was defined as HOMA $\geq 3.7 \mathrm{mmol}(\mu \mathrm{U}) / \mathrm{L}^{2}$ and EKD as stages 1 and 2 of the NKF-KDOQI. Carotid intima-media thickness was used as a surrogate marker of arteriosclerosis.

Results: The presence of one trait of metabolic syndrome was associated with an odds ratio (OR) for EKD of 2.3 (95\% confidence interval [CI], 1.18-4.48) that increased to 6.72 (95\% CI, 3.56-13.69) in subjects with the syndrome. All the traits of the syndrome except low level of high-density lipoproteins showed an increased OR for EKD. Increasing HOMA was also directly correlated with higher OR for EKD, being as high as 3.89 (95\% CI, 1.99-7.59) for subjects in the fourth quartile. Subjects with the syndrome plus EKD showed an increased intima-media thickness compared with those without kidney disease. Conclusions: Insulin resistance and all metabolic syndrome traits except low level of high-density lipoproteins were significantly associated with an increased OR for EKD. Both metabolic syndrome and EKD were independently and additively related to the presence of surrogate markers of arteriosclerosis.

Full English text available from: www.revespcardiol.org

(c) 2010 Sociedad Española de Cardiología. Published by Elsevier España, S.L. All rights reserved.

VÉASE CONTENIDO RELACIONADO:

DOI: 10.1016/j.recesp.2011.01.005, Rev Esp Cardiol 2011;64:358-60

* Autor para correspondencia: Departamento de Medicina Interna, Clínica Universidad de Navarra, Avda. Pío XII 36, 31008 Pamplona, Navarra, España.

Correo electrónico: mflandecho@unav.es (M.F. Landecho). 


\section{Abreviaturas}

CAC: cociente albúmina/creatinina

ER: enfermedad renal

ERP: enfermedad renal precoz

RI: resistencia a la insulina

SM: síndrome metabólico

\section{INTRODUCCIÓN}

La enfermedad renal (ER) avanzada y la terminal se han reconocido en los últimos años como importantes problemas de salud pública, dados su creciente prevalencia y su mal pronóstico ${ }^{1}$. Se sabe que, una vez iniciada, la ER tiende a progresar. Aun así, en los sujetos con ER avanzada las complicaciones cardiovasculares son más prevalentes que la progresión a ER terminal ${ }^{2,3}$. Mínimas reducciones del filtrado glomerular se asocian a un aumento del riesgo vascular ${ }^{4-8}$. Entre los factores asociados a la progresión de la ER, hay algunos susceptibles de intervención médica y otros que no. Entre los no controlables, destaca la edad $^{9-11}$. Los factores susceptibles de intervención médica son fundamentalmente los clásicos de riesgo vascular. Destacan la hipertensión arterial, la diabetes mellitus y la resistencia a la insulina (RI), la obesidad y la dislipemia, que en su conjunto conforman el síndrome metabólico (SM). La ER se divide en cinco estadios $^{1}$, agrupables en dos bloques. Por un lado, pacientes con datos analíticos de daño renal (microalbuminuria) y con filtrado glomerular $\geq 60 \mathrm{ml} / \mathrm{min} / 1,73 \mathrm{~m}^{2}$ (estadios 1 y 2 ; ER precoz [ERP]). Por otro lado, pacientes con filtrado glomerular patológico, independientemente de la presencia de microalbuminuria (estadios 3, 4 [ER avanzada] y 5 [ER terminal]).

El SM es una asociación de factores de riesgo para el que se ha descrito una mortalidad total y cardiovascular que aproximadamente duplica la de la población general ${ }^{12,13}$ pese a que el mayor conocimiento del cuadro ha conllevado mejoras significativas en el manejo individual de estos pacientes. Además, es una entidad de prevalencia creciente ${ }^{14-16}$. Entre los factores asociados al aumento de la prevalencia del SM, destacan el envejecimiento de la población y el sobrepeso ${ }^{16}$. Esta tendencia al aumento de la prevalencia, que ha alcanzado grado de pandemia ${ }^{17,18}$, se ha confirmado en nuestro país ${ }^{19-21}$. La RI es el defecto metabólico subyacente al SM.

La hipótesis de este trabajo es que el SM y sus componentes pueden asociarse a los estadios precoces de ER. Además, ambas entidades podrían tener un efecto sinérgico en el desarrollo de arteriosclerosis. Los objetivos son: el análisis de la posible relación global entre SM, sus componentes y la ERP; la valoración de la importancia de cada componente y de la RI en la presencia de ERP, y la valoración de la ERP y el SM como marcadores de riesgo cardiovascular independientes en el desarrollo de arteriosclerosis.

\section{MÉTODOS}

De acuerdo con las guías institucionales, los pacientes incluidos en el estudio fueron informados de su naturaleza investigadora y consintieron por escrito su participación. El estudio fue diseñado de acuerdo con la Declaración de Helsinki, y el protocolo fue aprobado por el Comité de Ética de la Clínica Universidad de Navarra. El estudio se llevó a cabo con sujetos que acudieron a la Unidad de Chequeos de la Clínica Universidad de Navarra para un reconocimiento médico habitual tras $12 \mathrm{~h}$ de ayuno.
La población se reclutó entre mayo de 1999 y enero de 2009. Se evaluó a 2.163 sujetos consecutivos a los que se realizó una revisión médica general. En todos los casos se recogió una historia clínica completa. Los criterios de exclusión fueron:

- Enfermedad arterial (claudicación de la marcha, cardiopatía isquémica o historia de accidente cerebrovascular) presente o pasada.

- Diagnóstico previo de hipertensión arterial y/o diabetes mellitus.

- Filtrado glomerular por debajo de $60 \mathrm{ml} / \mathrm{min} / 1,73 \mathrm{~m}^{2}$ (ER estadios 3 a 5).

Tras las exclusiones y la firma del consentimiento informado, 1.498 sujetos entraron a formar parte del estudio.

Utilizando los criterios establecidos por la National Kidney Foundation, se ha definido ERP como los estadios 1 y 2 de dicha clasificación. Para definir microalbuminuria, se determinó el cociente albúmina/creatinina (CAC) en una muestra de orina de micción aislada. Se definió daño renal como una microalbuminuria en el rango comprendido entre $17-300 \mathrm{mg} / \mathrm{g}$ para varones $\mathrm{y}$ $25-300 \mathrm{mg} / \mathrm{g}$ para mujeres ${ }^{1}$. Para estimar el filtrado glomerular, se usó la fórmula MDRD (Modification of Diet in Renal Disease) ${ }^{1}$.

Para el diagnóstico de SM, se han utilizado los criterios ATP-III modificados por la American Heart Association/National Heart, Lung, and Blood Institute (AHA/NHLBI) ${ }^{22}$. Se definió como población control a los sujetos con 0 criterios AHA/NHLBI.

Para cuantificar la RI, se ha utilizado el índice Homeostasis Model Assessment (HOMA). Sobre la base de un estudio preliminar, realizado sobre 50 sujetos control con índice de masa corporal $<25$, en el que el valor medio de HOMA fue $1,61 \pm 0,696 \mathrm{mmol}(\mu U) / \mathrm{l}^{2}$, se localizó el punto de corte de RI en HOMA $\geq 3,7 \mathrm{mmol}(\mu U) / 1^{2}$, correspondiente al valor medio de HOMA más 3 desviaciones estándar de dicha muestra. Dicho punto de corte coincide con publicaciones previas sobre población española ${ }^{23}$.

Se analizó el grosor íntima-media (GIM) carotídeo como marcador de arteriosclerosis subclínica en 1.000 de los 1.498 sujetos del estudio inicial. Todas las mediciones de GIM carotídeo se efectuaron mediante eco-Doppler, por ecografistas experimentados del Servicio de Radiología de nuestro centro. Las mediciones se hicieron con un transductor lineal de 5-12 MHz (ATL 500 HDI). Las variabilidades intraobservador e interobservador de los ecografistas ha sido validada en estudios previos de nuestro grupo $^{24-26}$. El punto de la medición fue $1 \mathrm{~cm}$ proximal del bulbo de cada arteria carótida común, en zonas libres de placa. El valor medio de ambos lados se utilizó como medida de GIM carotídeo. El punto de corte del GIM carotídeo patológico se situó en 0,75 mm. Se definió este punto por ser el límite inferior del cuartil superior de un estudio interno realizado en 156 sujetos controles sanos. Además, este punto de corte coincide con lo publicado para una muestra de población española de varones del mismo grupo de edad que la media de nuestro estudio ${ }^{27}$.

Las determinaciones analíticas se efectuaron mediante técnicas estándar de laboratorio.

Los resultados se expresaron como el valor de la media aritmética \pm desviación estándar de la media. En todos los casos se consideró que existían diferencias estadísticamente significativas para valores de $\mathrm{p}<0,05$.

Para analizar si una variable seguía una distribución normal dentro de una población, se utilizó el test de Shapiro-Wilks, Kolmogorov-Smirnov (con la corrección de Lliliefors) y para analizar la homogeneidad de varianzas entre dos muestras, el test de Lévene.

Para la comparación estadística de los parámetros cuantitativos entre tres o más grupos experimentales, se empleó el test ANOVA de un criterio para las muestras paramétricas, con el test $a$ 
posteriori de Tukey B o Scheffé. En el caso de muestras no paramétricas, se empleó el test de Kruskal-Wallis seguido del test U de Mann-Whitney para las comparaciones a posteriori.

Para la comparación de dos grupos experimentales, se utilizó el test de la t de Student para datos independientes, en el caso de muestras paramétricas, y el test U de Mann-Whitney en el caso de contar con muestras no paramétricas.

Para realizar los análisis bivariables, se utilizó el test de correlación de Pearson, mientras que el análisis multivariable se llevó a cabo mediante regresión lineal.

Los cálculos estadísticos se analizaron utilizando el programa estadístico SPSS versión 13.0.

\section{RESULTADOS}

La tabla 1 muestra las características generales de la población estudiada.

De los 1.498 sujetos estudiados, el 8,9\% cumplía criterios de ERP. En el análisis por grupos de edad, hallamos una prevalencia de ERP entre sujetos menores de 40 años del 2,7\%, que aumentó hasta el 8,8\% en el grupo de edad comprendido entre 40 y 60 años. Finalmente, fue del $13,1 \%$ en sujetos por encima de los 60 años. La tabla 2 compara las características de los sujetos según tuvieran SM o no. En la valoración de los sujetos sin SM, aquellos con 0 componentes de SM se diferenciaron de aquellos con 1 o 2 en que había un $74 \%$ de varones, frente a un $77 \%$ ( $p=0,04$ ); el índice de masa corporal fue inferior, pero en ambos casos en la franja de sobrepeso $(25,12 \pm 2,73$ frente a $26,91 \pm 3,62)$. Asimismo se hallaron diferencias en la glucemia y en la presión arterial sistólica, en ambos casos con valores dentro de la normalidad. El resto de las comparaciones no mostró diferencias significativas respecto a aquellos con 1 o 2 componentes del SM.

La prevalencia de ERP aumenta de forma progresiva $\mathrm{y}$ estadísticamente significativa conforme se acumulan componentes del SM. De los sujetos con 0 componentes, el 2,9\% cumplía criterios de ERP. Esta prevalencia aumentó hasta el 7,4\% con la aparición del primero, el 9,5\% con el segundo y el $20 \%$ con el diagnóstico de SM (p de tendencia $<0,001$ ). La tabla 3 muestra que,

Tabla 1

Características de la población general $(n=1.498)$

\begin{tabular}{|c|c|}
\hline Edad (años) & $51,63 \pm 10,42$ \\
\hline Varones, \% & 79,4 \\
\hline Fumadores, \% & 27,2 \\
\hline IMC & $27,63 \pm 3,99$ \\
\hline PAS (mmHg) & $122,06 \pm 18,47$ \\
\hline PAD (mmHg) & $79,25 \pm 9,49$ \\
\hline Glucemia (mg/dl) & $96,15 \pm 16,18$ \\
\hline Insulina $(\mathrm{mU} / \mathrm{l})$ & $10,57 \pm 7,54$ \\
\hline HOMA (mU*mmol/12) & $2,55 \pm 2,08$ \\
\hline Triglicéridos (mg/dl) & $115,14 \pm 73,77$ \\
\hline Colesterol total (mg/dl) & $223,57 \pm 41,02$ \\
\hline $\mathrm{HDL}(\mathrm{mg} / \mathrm{dl})$ & $52,55 \pm 14,71$ \\
\hline LDL (mg/dl) & $148,12 \pm 37,5$ \\
\hline Creatinina (mg/dl) & $0,93 \pm 0,15$ \\
\hline $\mathrm{ClCr}\left(\mathrm{ml} / \mathrm{min} / 1,73 \mathrm{~m}^{2}\right)$ & $87,68 \pm 14,79$ \\
\hline $\mathrm{CAC}(\mathrm{mg} / \mathrm{g})$ & $9,06 \pm 15,73$ \\
\hline
\end{tabular}

CAC: cociente albúmina/creatinina; $\mathrm{ClCr}$ : aclaramiento de creatinina; HDL: lipoproteínas de alta densidad; HOMA: Homeostasis Model Assessment; IMC: índice de masa corporal; LDL: lipoproteínas de baja densidad; PAD: presión arterial diastólica; PAS: presión arterial sistólica.

Los resultados expresan el valor de la media aritmética \pm desviación estándar de la media, salvo otra indicación.
Tabla 2

Características de la población según la presencia de síndrome metabólico

\begin{tabular}{|c|c|c|c|}
\hline & Sin SM & Con SM & $\mathrm{p}$ \\
\hline Pacientes & 1.208 & 290 & \\
\hline Edad (años) & $51,24 \pm 10,51$ & $53,19 \pm 9,91$ & 0,001 \\
\hline Varones, \% & 76,8 & 90,7 & $<0,001$ \\
\hline Fumadores, \% & 25,5 & 34,5 & 0,002 \\
\hline IMC & $26,7 \pm 3,45$ & $31,51 \pm 3,73$ & $<0,001$ \\
\hline PAS (mmHg) & $118,88 \pm 17,07$ & $135,28 \pm 18,25$ & $<0,001$ \\
\hline PAD (mmHg) & $77,67 \pm 8,8$ & $85,85 \pm 9,42$ & $<0,001$ \\
\hline Glucemia (mg/dl) & $93,32 \pm 11,32$ & $107,96 \pm 25,44$ & $<0,001$ \\
\hline Insulina $(\mathrm{mU} / \mathrm{l})$ & $9,1 \pm 6,03$ & $17,37 \pm 9,76$ & $<0,001$ \\
\hline HOMA $\left(\mathrm{mU} \times \mathrm{mmol} / \mathrm{l}^{2}\right)$ & $2,1 \pm 1,52$ & $4,63 \pm 2,88$ & $<0,001$ \\
\hline Triglicéridos (mg/dl) & $98,34 \pm 51,45$ & $184,85 \pm 105,2$ & $<0,001$ \\
\hline Colesterol total $(\mathrm{mg} / \mathrm{dl})$ & $221,32 \pm 39,68$ & $232,94 \pm 45,03$ & $<0,001$ \\
\hline HDL (mg/dl) & $54,83 \pm 14,68$ & $43,03 \pm 10,34$ & $<0,001$ \\
\hline LDL (mg/dl) & $146,77 \pm 36,79$ & $153,8 \pm 39,93$ & 0,008 \\
\hline Creatinina (mg/dl) & $0,93 \pm 0,15$ & $0,94 \pm 0,13$ & 0,241 \\
\hline $\mathrm{ClCr}\left(\mathrm{ml} / \mathrm{min} / 1,73 \mathrm{~m}^{2}\right)$ & $87,51 \pm 14,85$ & $88,43 \pm 14,54$ & 0,286 \\
\hline CAC $(\mathrm{mg} / \mathrm{g})$ & $7,82 \pm 13,38$ & $14,24 \pm 22,36$ & $<0,001$ \\
\hline CAC patológico, \% & 6,3 & 20 & $<0,001$ \\
\hline
\end{tabular}

CAC: cociente albúmina/creatinina; $\mathrm{ClCr}$ : aclaramiento de creatinina; $\mathrm{HDL}$ lipoproteínas de alta densidad; HOMA: Homeostasis Model Assessment; IMC: índice de masa corporal; LDL: lipoproteínas de baja densidad; PAD: presión arterial diastólica; PAS: presión arterial sistólica; SM: síndrome metabólico.

Los resultados expresan el valor de la media aritmética \pm desviación estándar de la media, salvo otra indicación.

en relación con controles sanos, el riesgo de ERP estimado mediante odds ratio (OR) aumenta en relación con el número de componentes de SM.

La figura 1 muestra el incremento significativo de la prevalencia de ERP con la edad y según la ausencia o presencia de SM, que alcanza al $26,3 \%$ de los sujetos de mayor edad y con SM.

La tabla 4 muestra que la RI y todos los componentes del SM, excepto un valor bajo de lipoproteínas de alta densidad (HDL), se asocian de forma individualmente significativa con un mayor riesgo de ERP.

En la medida en que aumenta el grado de RI, se incrementa la prevalencia de ERP, desde el 4,2\% en el primer cuartil hasta el 4,5\% en el segundo; el 10,2\% en el tercero y el $15,7 \%$ en el cuarto cuartil ( $\mathrm{p}$ de tendencia $<0,001$ ). La tabla 5 muestra que el riesgo de ERP estimado mediante OR aumenta en relación directa con el grado de RI clasificado en cuartiles de HOMA.

La presencia de SM se asoció con un mayor GIM carotídeo, que pasó desde $0,61 \pm 0,008 \mathrm{~mm}$ en sujetos control hasta $0,71 \pm 0,009 \mathrm{~mm}$ en pacientes con SM ( $\mathrm{p}<0,001$ ). Por otro lado, respecto a los mismos controles sanos, la presencia de ERP también se asoció con un aumento significativo del GIM carotídeo, que fue $0,75 \pm 0,01 \mathrm{~mm}(\mathrm{p}<0,001)$. La figura 2 muestra que, en sujetos con SM, la coexistencia de ERP se asoció con un GIM carotídeo significativamente mayor ( $p=0,006)$.

La tabla 6 muestra el comportamiento del SM y la ERP como marcadores de riesgo independientes de GIM carotídeo patológico estimado mediante el cálculo de la OR.

Se valoró la OR del GIM carotídeo patológico de la ERP y cada componente del SM. En un análisis ajustado por edad, sexo, ERP y los componentes de SM, sólo la ERP ( $\mathrm{OR}=1,65$; intervalo de confianza [IC] del 95\%, 1,01-2,69; $\mathrm{p}=0,044)$, la presión arterial elevada (OR = 1,6; IC del 95\%, 1,18-2,17; $\mathrm{p}=0,003)$ y el incremento de perímetro abdominal $(\mathrm{OR}=1,44$; IC del 95\%, 1,03-2,03; $\mathrm{p}=0,032$ ) se comportaron como marcadores de riesgo independiente de GIM carotídeo patológico. 
Tabla 3

Estimación del riesgo de enfermedad renal precoz según el número de componentes de síndrome metabólico

\begin{tabular}{|c|c|c|c|c|}
\hline \multirow[t]{2}{*}{ Componentes } & \multicolumn{4}{|c|}{ OR (IC del 95\%) } \\
\hline & No ajustado & $\mathrm{p}$ & Ajustado por edad y sexo & $\mathrm{p}$ \\
\hline 1 & $2,7(1,39-5,22)$ & 0,003 & $2,3(1,18-4,48)$ & 0,014 \\
\hline 2 & $3,5(1,8-6,81)$ & $<0,001$ & $2,98(1,51-5,9)$ & 0,002 \\
\hline 3 o más & $8,4(4,51-15,65)$ & $<0,001$ & $6,72(3,56-12,69)$ & $<0,001$ \\
\hline
\end{tabular}

IC: intervalo de confianza; OR: odds ratio.

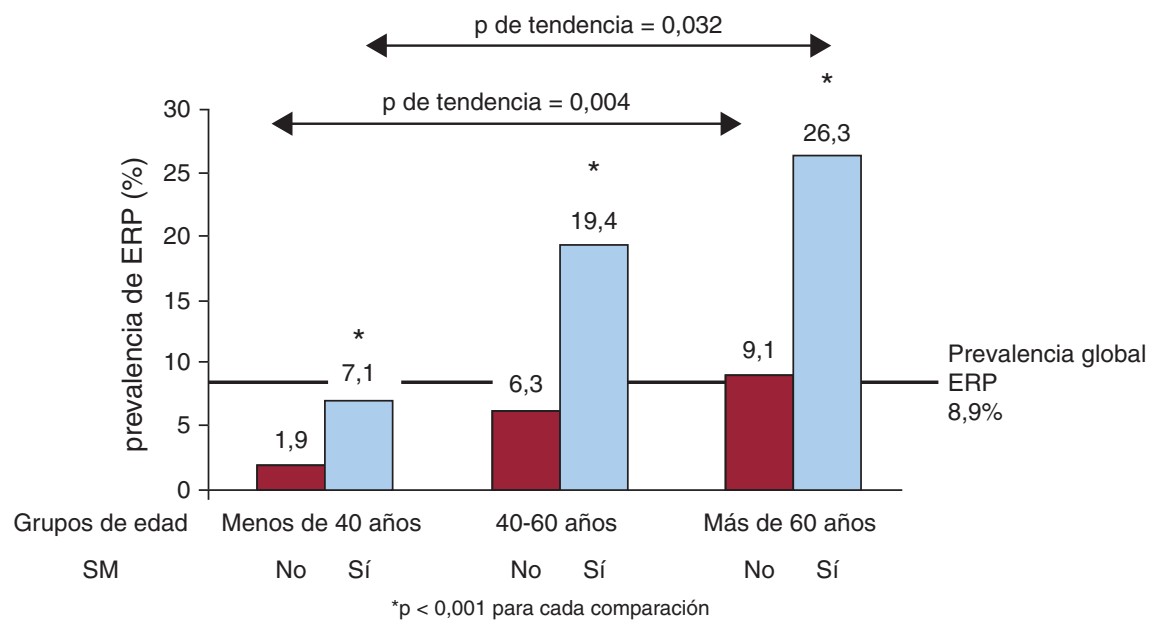

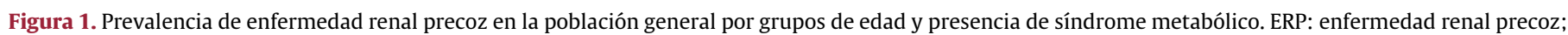
SM: síndrome metabólico.

\section{DISCUSIÓN}

Los resultados del presente trabajo confirmaron que la ERP es una entidad cuya frecuencia aumenta con la edad, ya que la prevalencia es cerca de 5 veces superior en los sujetos mayores de 60 años que en los menores de 40 . Este deterioro de la función renal con la edad coincide con datos disponibles en la literatura ${ }^{9,10}$. Según el registro EPIRCE (Epidemiología de la Insuficiencia Renal Crónica en España), la prevalencia de ERP en nuestro país podría rondar el $2,2 \%$, en una población con una media de edad de
$49 \mathrm{años}^{28}$. Los datos del presente trabajo indicarían una prevalencia muy superior pero, dadas las características del muestreo, no es posible hacer estimaciones poblacionales de prevalencia. En cualquier caso, se confirma que la ERP es una entidad frecuente en nuestro entorno, sobre todo entre sujetos de mayor edad.

La prevalencia de ERP en sujetos con SM fue significativamente superior que entre sujetos sin SM. Además, el riesgo de ERP se duplicó desde la aparición de un criterio de SM, y aumentó progresivamente hasta ser 6,72 veces superior en los sujetos con

Tabla 4

Estimación del riesgo (odds ratio) de enfermedad renal precoz según el componente específico de síndrome metabólico

\begin{tabular}{|c|c|c|c|c|}
\hline \multirow[t]{2}{*}{ Factor } & \multicolumn{4}{|c|}{ OR (IC del 95\%) } \\
\hline & No ajustado & $\mathrm{p}$ & Ajustado por edad y sexo & $\mathrm{p}$ \\
\hline Obesidad & $2,54(1,76-3,65)$ & $<0,001$ & $2,31(1,6-3,34)$ & $<0,001$ \\
\hline Tg-e & $3,26(2,25-4,71)$ & $<0,001$ & $3,21(2,19-4,68)$ & $<0,001$ \\
\hline HDL-b & $1,11(0,72-1,72)$ & 0,616 & $1,14(0,73-1,77)$ & 0,543 \\
\hline Glu-e & $2,12(1,48-3,04)$ & $<0,001$ & $1,73(1,19-2,51)$ & $<0,004$ \\
\hline Pa-e & $3,43(2,34-5,02)$ & $<0,001$ & $2,94(1,98-4,35)$ & $<0,001$ \\
\hline RI & $2,58(1,68-3,98)$ & $<0,001$ & $2,37(1,53-3,67)$ & $<0,001$ \\
\hline
\end{tabular}

Glu-e: glucemia basal elevada según criterio de síndrome metabólico; HDL-b: valor bajo de lipoproteínas de alta densidad según criterio de síndrome metabólico; IC: intervalo de confianza; OR: odds ratio; Pa-e: presión arterial elevada según criterio de síndrome metabólico; RI: resistencia a la insulina; Tg-e: triglicéridos elevados según criterio de síndrome metabólico.

Tabla 5

Estimación del riesgo de enfermedad renal precoz según el grado de resistencia a la insulina calculado en cuartiles del índice Homeostasis Model Assessment

\begin{tabular}{llcc}
\hline Factor & \multicolumn{2}{c}{ OR (IC del 95\%) } \\
\cline { 2 - 4 } & No ajustado & $\mathrm{p}$ & Ajustado por edad y sexo \\
\hline Segundo cuartil & $1,08(0,48-2,42)$ & 0,838 & $0,99(0,44-2,23)$ \\
\hline Tercer cuartil & $2,62(1,31-5,23)$ & 0,006 & $2,55(1,26-5,15)$ \\
\hline Cuarto cuartil & $4,29(2,22-8,3)$ & $<0,001$ & $3,89(1,99-7,59)$ \\
\hline
\end{tabular}

IC: intervalo de confianza; OR: odds ratio; primer cuartil: $<1,21(\mathrm{mU} \times \mathrm{mmol}) / \mathrm{I}^{2}$; segundo cuartil: $1,21-2,01(\mathrm{mU} \times \mathrm{mmol}) / \mathrm{I}^{2}$; tercer cuartil: $2,02-3,21(\mathrm{mU} \times \mathrm{mmol}) / \mathrm{I}^{2}$; cuarto cuartil: > 3,21 $(\mathrm{mU} \times \mathrm{mmol}) / 1^{2}$. 
Tabla 6

Estimación del riesgo de grosor íntima-media carotídeo patológico según síndrome metabólico y daño renal

\begin{tabular}{|c|c|c|c|c|c|c|}
\hline \multirow[t]{2}{*}{ Factor } & \multicolumn{6}{|c|}{ OR (IC del 95\%) } \\
\hline & No ajustado & $\mathrm{p}$ & Ajustado por edad y sexo & $\mathrm{p}$ & Ajuste multivariable* & $\mathrm{p}$ \\
\hline SM & $1,83(1,33-2,52)$ & $<0,001$ & $1,78(1,21-2,63)$ & 0,001 & $1,53(1,07-2,85)$ & 0,024 \\
\hline ERP & $2,42(1,56-3,65)$ & $<0,001$ & $1,95(1,19-3,21)$ & 0,008 & $1,75(1,07-2,85)$ & 0,032 \\
\hline
\end{tabular}

ERP: enfermedad renal precoz; IC: intervalo de confianza; OR: odds ratio; SM: síndrome metabólico.

*Ajustado por edad, sexo y SM o ERP.

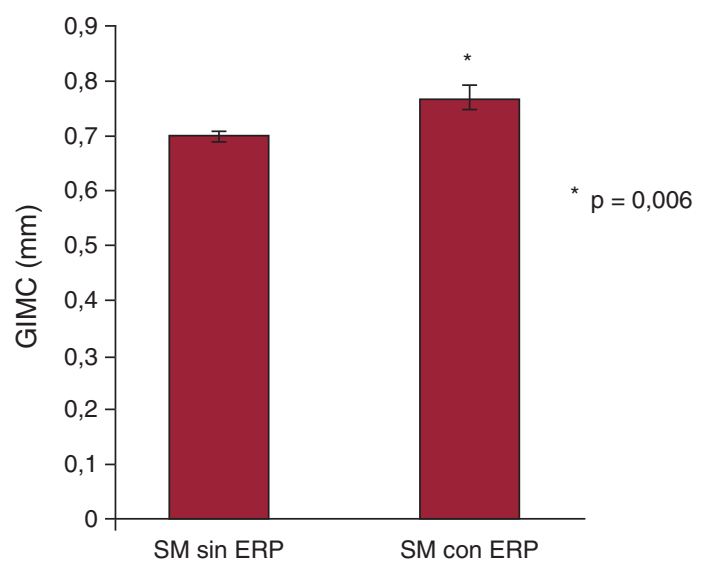

Figura 2. Grosor íntima-media carotídeo en pacientes con síndrome metabólico sin y con enfermedad renal precoz. ERP: enfermedad renal precoz; GIMC: grosor íntima-media carotídeo; SM: síndrome metabólico.

tres o más componentes. Estos datos coinciden con lo descrito previamente para la ER avanzada, con un exceso de riesgo entre 1,4 y $2,6^{29-32}$. Que nosotros sepamos, no hay datos sobre la asociación entre la ERP y el SM. En todos los grupos de edad, la prevalencia de ERP fue notablemente superior en sujetos con SM. Además, la diferencia entre ambas prevalencias es más marcada en cada grupo de edad respecto al previo. Cabe destacar que la prevalencia de ERP en sujetos mayores de 60 años con SM fue del 26,3\%, lo que indica un efecto sinérgico de la edad y el SM para el deterioro de la función renal. Por lo tanto, y dada la alta prevalencia de SM en España ${ }^{19-21}$, consideramos que se podría seleccionar a un gran número de pacientes con alto riesgo de ERP en los que hacer determinación combinada de filtrado glomerular y CAC.

En la valoración de cada componente de SM, se halló que todos, salvo el valor bajo de HDL, se asociaron con una OR elevada de ERP. Aunque el valor bajo de HDL es un factor de riesgo bien contrastado para el deterioro del filtrado glomerular, su asociación con la presencia de microalbuminuria no está demostrada ${ }^{29,30,33-37}$.

La presencia de cifras elevadas de triglicéridos triplicó el riesgo de ERP. Esta asociación esta descrita para la ER avanzada, con una OR de entre 1,34 y $1,65^{29,30,33,34}$.

Estos resultados mostraron que la obesidad duplica el riesgo de ERP. La obesidad se ha asociado con un aumento de riesgo de desarrollar ER avanzada o terminal con un incremento de riesgo entre 1,73 y 3,57 según los estudios consultados ${ }^{29,38}$.

Según los datos de este estudio, con el punto de corte de glucemia en $100 \mathrm{mg} / \mathrm{dl}$, los sujetos con glucemia basal elevada tienen un riesgo de ERP 1,73 veces superior que los sujetos normoglucémicos. En otros estudios que han utilizado el criterio de glucemia basal de $110 \mathrm{mg} / \mathrm{dl}$, no se detectó relación entre este y las fases avanzadas de la $\mathrm{ER}^{29,30}$. Estos resultados parecen indicar que mínimas elevaciones de la glucemia se asocian significativamente a deterioro inicial de la función renal.

En la muestra del presente estudio, los sujetos que cumplían los criterios AHA/NHLBI de presión arterial elevada tenían un riesgo estimado de ERP 2,94 veces superior. Esta observación coincide con la literatura, ya que actualmente se considera que las cifras de presión arterial son un factor de riesgo continuo en lo que a la progresión de la disfunción renal se refiere ${ }^{39}$. Estos resultados confirman que mínimas alteraciones de la presión arterial se asocian significativamente con el deterioro incipiente de la función renal.

Los resultados del presente trabajo indican que existe una relación significativa entre el grado de RI y la prevalencia de ERP. En la medida que aumenta el valor de HOMA, se produce un incremento de la prevalencia de ERP desde el 4,2\% en el primer cuartil hasta el 15,7\% en el cuarto. Además, el riesgo de ERP en sujetos con RI estaba significativamente aumentado respecto a los sujetos sensibles a la insulina. La relación entre la ER avanzada y la RI se intuye en algunos estudios poblacionales ${ }^{31,40}$, pero no se conocía como tal su relación con la ERP.

El GIM carotídeo es un modo útil y no invasivo de evaluación de arteriosclerosis carotídea precoz, hasta el punto que se lo considera un predictor de eventos vasculares ${ }^{41,42}$. Los datos del presente estudio muestran que los pacientes con ERP presentan un aumento significativo del GIM carotídeo respecto a los controles sanos. En el análisis multivariable ajustado por edad y sexo, la ERP se comportó como un marcador de riesgo independiente en el desarrollo de arteriosclerosis subclínica incluso tras ajustar por SM. Estudios preliminares de nuestro grupo habían indicado que la ERP podría asociarse a un GIM carotídeo aumentado ${ }^{43}$. Estos resultados refuerzan el concepto de que la ER, incluso en fases iniciales, potencia la enfermedad arterial mucho antes de que se produzca clínica asociada al deterioro renal ${ }^{4,44}$. Además, estos resultados muestran que el GIM carotídeo de los sujetos control era significativamente inferior que el de los sujetos con SM sin ERP, y este, a su vez, menor que el de los casos con SM y ERP. Este dato podría indicar un efecto sinérgico de SM y ERP en el desarrollo de arteriosclerosis subclínica. Los datos del presente estudio indican que los factores más estrechamente asociados al desarrollo de arteriosclerosis subclínica en el contexto del SM son la obesidad y la presión arterial elevada, pero que incluso por encima de ellas se encuentra la ERP, como muestran las diferencias entre las OR halladas.

\section{Implicaciones clínicas}

En consecuencia, el cribado de la ERP puede ser de gran ayuda en la valoración del riesgo cardiovascular en pacientes con el SM. Nuestros resultados sientan las bases de estudios prospectivos que permitan evaluar el efecto de la prevención y el tratamiento individual de cada componente del SM en la iniciación y la progresión de las enfermedades arteriosclerótica y renal.

\section{Limitaciones}

Entre las limitaciones de este estudio, hemos detectado un posible sesgo de clasificación de los sujetos con o sin ERP, ya que contamos con una única estimación del filtrado glomerular y una determinación de CAC. La determinación urinaria de albúmina puede verse afectada por múltiples factores (hipertensión arterial mal controlada, ejercicio físico en las $24 \mathrm{~h}$ anteriores, dieta hiperproteica y otras), por lo que se recomienda su repetición al 
menos dos o tres veces en el plazo de 3-6 meses. En caso de que se hubiera cometido este error, se habría clasificado erróneamente a alguno de los participantes como ERP sí/no. En consecuencia, se habría dificultado el hallazgo de diferencias entre ambos grupos, ya que este sesgo tendería al valor nulo. Por lo tanto, es de suponer que una clasificación más estricta de la población mostrase datos aún más contundentes que los expuestos.

Una publicación reciente ${ }^{45}$ señala que el sexo podría ser un factor importante en la aparición de ER estadios 1 a 5 en los pacientes con SM. Esta asociación no se ha valorado en el presente estudio, por lo que estudios futuros podrían matizar los resultados aquí expuestos.

No se dispone de marcadores validados para describir el nivel socioeconómico de la población del estudio.

\section{CONCLUSIONES}

El SM es un importante marcador de riesgo de ERP. Coherentemente, su sustrato fisiopatológico, la RI, y todos sus componentes, salvo el valor bajo de HDL, se asocian con una mayor prevalencia de ERP. Por otro lado, en los pacientes con SM la coexistencia de ERP aumenta significativamente el riesgo de un GIM carotídeo engrosado respecto a aquellos sin ERP.

\section{CONFLICTO DE INTERESES}

Ninguno.

\section{BIBLIOGRAFÍA}

1. Levey AS, Coresh J, Balk E, Kausz AT, Levin A, Steffes MW, et al. National Kidney Foundation Practice Guidelines for Chronic Kidney Disease: Evaluation, Classification, and Stratification. Ann Intern Med. 2003;139:137-47.

2. Desbien AM, Chonchol M, Gnahn H, Sander D. Kidney function and progression of carotid intima-media thickness in a community study. Am J Kidney Dis. 2008;51:584-93.

3. Manjunath G, Tighiouart H, Coresh J, Macleod B, Salem DN, Griffith JL, et al. Level of kidney function as a risk factor for cardiovascular outcomes in the elderly. Kidney Int. 2003;63:1121-9.

4. Sarnak MJ, Levey AS, Schoolwerth AC, Coresh J, Culleton B, Hamm LL, et al. Kidney disease as a risk factor for development of cardiovascular disease: A statement from the American Heart Association Councils on Kidney in Cardiovascular Disease, High Blood Pressure Research, Clinical Cardiology, and Epidemiology and Prevention. Circulation. 2003;108:2154-69.

5. Ritz E. Minor renal dysfunction: an emerging independent cardiovascular risk factor. Heart. 2003;89:963-4.

6. Ruilope LM, Van Veldhuisen DJ, Ritz E, Luscher TF. Renal function: the Cinderella of cardiovascular risk profile. J Am Coll Cardiol. 2001;38:1782-7.

7. Ritz E. Heart and kidney: fatal twins? Am J Med. 2006;119 5 Suppl 1:31-9.

8. Go AS, Chertow GM, Fan D, McCulloch CE, Hsu Cy. Chronic kidney disease and the risks of death, cardiovascular events, and hospitalization. N Engl J Med. 2004;351:1296-305.

9. Lindeman RD, Tobin J, Shock NW. Longitudinal studies on the rate of decline in renal function with age. J Am Geriatr Soc. 1985;33:278-85.

10. Stevens LA, Coresh J, Greene T, Levey AS. Assessing kidney function-measured and estimated glomerular filtration rate. N Engl J Med. 2006;354:2473-83.

11. Fox CS, Larson MG, Leip EP, Culleton B, Wilson PW, Levy D. Predictors of new-onset kidney disease in a community-based population. JAMA. 2004;291:844-50.

12. Dekker JM, Girman C, Rhodes T, Nijpels G, Stehouwer CD, Bouter LM, et al. Metabolic syndrome and 10-year cardiovascular disease risk in the Hoorn Study. Circulation. 2005;112:666-73.

13. Galassi A, Reynolds K, He J. Metabolic syndrome and risk of cardiovascular disease: a meta-analysis. Am J Med. 2006;119:812-9.

14. Ford ES, Giles WH, Dietz WH. Prevalence of the metabolic syndrome among US adults: Findings from the Third National Health and Nutrition Examination Survey. JAMA. 2002;287:356-9.

15. Ford ES. Prevalence of the metabolic syndrome defined by the International Diabetes Federation among adults in the U.S. Diabetes Care. 2005;28:2745-9.

16. Park YW, Zhu S, Palaniappan L, Heshka S, Carnethon MR, Heymsfield SB. The metabolic syndrome: prevalence and associated risk factor findings in the US population from the Third National Health and Nutrition Examination Survey, 1988-1994. Arch Intern Med. 2003;163:427-36.

17. Cameron AJ, Shaw JE, Zimmet PZ. The metabolic syndrome: prevalence in worldwide populations. Endocrinol Metab Clin North Am. 2004;33:351-75.
18. Grundy SM. Metabolic syndrome pandemic. Arterioscler Thromb Vasc Biol. 2008;28:629-36.

19. Alegría E, Cordero A, Laclaustra M, Grima A, León M, Casasnovas JA, et al. Prevalencia del síndrome metabólico en población laboral española: registro MESYAS. Rev Esp Cardiol. 2005;58:797-806.

20. Coca Díaz MM, Hernanz LP, Vega GM, Suárez FC. Prevalencia del síndrome metabólico en pacientes de un área urbana de atención primaria. Aten Primaria. 2005;35:436.

21. Viñes JJ, Díez J, Guembe MJ, González P, Amezqueta C, Barba J, et al. Estudio de riesgo vascular en Navarra: objetivos y diseño. Prevalencia del síndrome metabólico y de los factores mayores de riesgo vascular. An Sist Sanit Navar. 2007;30:113-24.

22. Grundy SM, Cleeman JI, Daniels SR, Donato KA, Eckel RH, Franklin BA, et al. Diagnosis and management of the metabolic syndrome. An American Heart Association/National Heart, Lung, and Blood Institute Scientific Statement. Circulation. 2005;112:2735-52.

23. Ascaso JF, Romero P, Real JT, Priego A, Valdecabres C, Carmena R. Cuantificación de insulinorresistencia con los valores de insulina basal e índice HOMA en una población no diabética. Med Clin (Barc). 2001;117:530-3.

24. Beloqui O, Páramo JA, Orbe J, Benito A, Colina I, Monasterio A, et al. Monocyte cyclooxygenase-2 overactivity: a new marker of subclinical atherosclerosis in asymptomatic subjects with cardiovascular risk factors? Eur Heart J. 2005;26:153-8.

25. Zalba G, Beloqui O, San José G, Moreno MU, Fortuño A, Díez J. NADPH oxidasedependent superoxide production is associated with carotid intima-media thickness in subjects free of clinical atherosclerotic disease. Arterioscler Thromb Vasc Biol. 2005;25:1452-7.

26. Páramo JA, Orbe J, Beloqui O, Benito A, Colina I, Martínez-Vila E, et al. Prothrombin fragment $1+2$ is associated with carotid intima-media thickness in subjects free of clinical cardiovascular disease. Stroke. 2004;35:1085-9.

27. Junyent M, Gilabert R, Núñez I, Corbella E, Vela M, Zambón D, et al. Ecografía carotídea en la evaluación de aterosclerosis preclínica. Distribución de valores del grosor íntima-media y frecuencia de placas de ateroma en una cohorte comunitaria española. Med Clin (Barc). 2005;125:770-4.

28. Otero A, De Francisco AL, Gayoso P, García F. Prevalencia de la enfermedad renal crónica en España: resultados del estudio EPIRCE. Nefrologia. 2010;1:78-86.

29. Chen J, Muntner P, Hamm LL, Jones DW, Batuman V, Fonseca V, et al. The metabolic syndrome and chronic kidney disease in U.S. adults. Ann Intern Med. 2004;140:167-74

30. Kurella M, Lo JC, Chertow GM. Metabolic syndrome and the risk for chronic kidney disease among nondiabetic adults. J Am Soc Nephrol. 2005;16:2134-40.

31. Tanaka H, Shiohira Y, Uezu Y, Higa A, Iseki K. Metabolic syndrome and chronic kidney disease in Okinawa. Japan Kidney Int. 2006;69:369-74.

32. Ninomiya T, Kiyohara Y, Kubo M, Yonemoto K, Tanizaki Y, Doi Y, et al. Metabolic syndrome and CKD in a general Japanese population: the Hisayama Study. Am J Kidney Dis. 2006;48:383-91.

33. Muntner P, Coresh J, Smith JC, Eckfeldt J, Klag MJ. Plasma lipids and risk of developing renal dysfunction: the atherosclerosis risk in communities study. Kidney Int. 2000;58:293-301.

34. Tozawa M, Iseki K, Iseki C, Oshiro S, Ikemiya Y, Takishita S. Triglyceride, but not total cholesterol or low-density lipoprotein cholesterol levels, predict development of proteinuria. Kidney Int. 2002;62:1743-9.

35. Stehouwer CD, Smulders YM. Microalbuminuria and risk for cardiovascular disease: Analysis of potential mechanisms. J Am Soc Nephrol. 2006;17: 2106-11.

36. Smulders YM, Rakic M, Stehouwer CD, Weijers RN, Slaats EH, Silberbusch J. Determinants of progression of microalbuminuria in patients with NIDDM. A prospective study. Diabetes Care. 1997;20:999-1005.

37. Cirillo M, Senigalliesi L, Laurenzi M, Alfieri R, Stamler J, Stamler R, et al. Microalbuminuria in nondiabetic adults: relation of blood pressure, body mass index, plasma cholesterol levels, and smoking: The Gubbio Population Study. Arch Intern Med. 1998;158:1933-9.

38. Hsu CY, McCulloch CE, Iribarren C, Darbinian J, Go AS. Body mass index and risk for end-stage renal disease. Ann Intern Med. 2006;144:21-8.

39. Forman JP, Brenner BM. 'Hypertension' and 'microalbuminuria': the bell tolls for thee. Kidney Int. 2006;69:22-8.

40. Kubo M, Kiyohara Y, Kato I, Iwamoto H, Nakayama K, Hirakata H, et al. Effect of hyperinsulinemia on renal function in a general Japanese population: the Hisayama study. Kidney Int. 1999;55:2450-6.

41. Lorenz MW, Markus HS, Bots ML, Rosvall M, Sitzer M. Prediction of clinical cardiovascular events with carotid intima-media thickness: a systematic review and meta-analysis. Circulation. 2007;115:459-67.

42. De Groot E, Hovingh GK, Wiegman A, Duriez P, Smit AJ, Fruchart JC, et al. Measurement of arterial wall thickness as a surrogate marker for atherosclerosis. Circulation. 2004;109 23 Suppl 1:33-8.

43. Fortuño A, Beloqui O, San José G, Moreno MU, Zalba G, Díez J. Increased phagocytic nicotinamide adenine dinucleotide phosphate oxidase-dependent superoxide production in patients with early chronic kidney disease. Kidney Int Suppl. 2005;99:S71-5.

44. Vanholder R, Massy Z, Argiles A, Spasovski G, Verbeke F, Lameire N. Chronic kidney disease as cause of cardiovascular morbidity and mortality. Nephrol Dial Transplant. 2005;20:1048-56.

45. Yu M, Ryu DR, Kim SJ, Choi KB, Kang DH. Clinical implication of metabolic syndrome on chronic kidney disease depends on gender and menopausal status: results from the Korean National Health and Nutrition Examination Survey. Nephrol Dial Transplant. 2010;25:469-77. 